\title{
SOM-Based Approach for the Analysis and Classification of Synchronous Impulsive Noise of an In-Ship PLC System
}

\author{
G. Acciani, V. Amoruso, G. Fornarelli, and A. Giaquinto \\ Dipartimento di Elettrotecnica ed Elettronica, Politecnico di Bari, Via E. Orabona 4, 70125 Bari, Italy \\ Correspondence should be addressed to A. Giaquinto, a.giaquinto@deemail.poliba.it
}

Received 24 July 2012; Accepted 10 September 2012

Academic Editors: C. Kotropoulos and W. Lam

Copyright () 2012 G. Acciani et al. This is an open access article distributed under the Creative Commons Attribution License, which permits unrestricted use, distribution, and reproduction in any medium, provided the original work is properly cited.

\begin{abstract}
The interest in wideband data transmission over power line communications has increased rapidly. This technology offers a convenient and inexpensive medium to transmit data, reducing the number of cables. This advantage is particularly appealing in many fields, like the railway, naval, and aeronautical ones. Nevertheless, several problems have to be faced to obtain a high data rate. In particular, the presence of noise makes the transmission difficult, degrading the quality of received signals and prohibiting the full application of these communication frameworks. In this paper the behaviour of an in-ship powerline communication system is analyzed in the presence of synchronous periodic impulsive noise. Such noise is modelled at source and its effects on the transmission of wideband signals are evaluated by means of a simulation circuit model. The obtained results allow to identify the characteristics of the channel and the critical conditions due to noise. Subsequently, an unsupervised technique based on principal component analysis and fuzzy $c$-mean classifier detects the presence and classifies the specific noises. Numerical results show that the proposed approach enables to achieve this target accurately under different operating conditions, proving to be an effective tool to enhance the performances of the considered technology.
\end{abstract}

\section{Introduction}

In recent years, interest in wideband data transmission over power line communications (PLCs) has been growing deeply $[1,2]$. PLCs represent a convenient and inexpensive solution for data transmission, since they are based on the idea of transmitting data by making use of media that was originally designed for the electrical energy delivery. This advantage is particularly welcome in the field of mobile vehicles, like aircraft and ships, in which the problem of sizes and weight of cables is felt significantly. Nevertheless, several problems have to be solved to obtain a high data rate transmission system. A first problem is due to the fact that the load impedances, which are distributed on the network, produce multiple reflections. Therefore, the structure of the powerline constitutes a time dependent and reverberating system. Moreover, the network configuration varies suddenly whenever an electric device is plugged in or out. Further factors make the data transmission difficult, in particular the presence of noise causes the decay of the performances [3]. Noise influences the transmission quality severely, in fact in PLCs impulsive phenomena occur, overlapping to a background noise. In addition, impulsive noises cannot be represented by simple additive models as for the classic transmission channels. Therefore, the modelling of noise and the study of its consequences on the bit error have attracted the attention of many researchers [4-6]. An effective classification of noises in PLCs is given in [3] and is the most commonly accepted [7]. According to the proposed scheme noises can be classified as belonging to the following classes: (a) coloured background noise; (b) narrow band noise; (c) periodic impulsive noise asynchronous to the main frequency; (d) periodic impulsive noise synchronous to the main frequency; (e) asynchronous impulsive noise. The cited classes of noises can be caused by a wide set of events. In detail, class (a) is caused by the summation of numerous noise sources of low power, class (b) is generated by the presence of radio broadcasting stations, and class (c) is caused by switched-mode power supplies. Lastly, classes (d) and (e) are due to the switching of rectifier diodes and the switching transients in the power network.

The classification of noisy events plays a fundamental role in PLCs applications. In particular, the capability of 
automatic recognizing of noises allows to minimize their effects in transmitting data [5]. To this aim, both supervised approaches and unsupervised ones can be taken into account. The former aim at classifying data as belonging to a set of classes which are known a priori; on the other hand the latter ones partition data on the basis of their observations, that is, their structure. Therefore, an unsupervised approach proves to be more flexible to detect the occurring of a noisy event [8]. In fact, the characteristics of impulsive noise at the receiver depend on the specific kinds of devices which are connected to the electric network and removed from it during the transmission.

The employment of unsupervised techniques requires a proper database to be constructed by means of actual measurements in order to synthesize the classifiers correctly. Nevertheless, this approach is not practical, in fact, this process could be very expensive, complex, and time consuming. An alternative way consists of obtaining the required data by using a simulative analysis. To this purpose, in previous works an additive model was mainly considered to take into account the effects of both background and impulsive events $[9,10]$. Since this kind of noise depends on the specific appliance which produces it, an alternative approach consists of modelling the disturbances at source as recently proposed in $[2,4]$. This approach allows to separate the modelling of noise from the modelling of the channel. In this way, the classification is made more feasible, in fact the noise signatures are correlated only with the sources. In this scheme the effects of the channel are taken into account, since noise at receiver would correspond to the noise model at source filtered by the powerline channel.

On the basis of these considerations, in this paper, the performances of a PLC system in the presence of six classes of impulsive noises are analyzed. Such kind of noises are generated by the transitions between $\mathrm{OFF} / \mathrm{ON}$ and vice versa states of some common appliances. Moreover, they are synchronous impulsive noise modelled at source as in [4]. The considered study case corresponds to a channel used to transmit wideband signals on a ship. This choice is due to the fact that the presence of a great amount of both ordinary electrical loads, like security systems, safety devices, and pumps, and special ones, such as on-board entertainment, devices for wide band internet connection, and control systems, characterizes modern ships and yachts. The set of these electrical loads makes the electrical system very complex. Therefore, this environment needs to be carefully investigated in order to evaluate its performances when working as a PLC system $[11,12]$. In detail, a reduced electrical scheme of a ship, in which the most representative loads are taken into account, is studied. The analysis conducted in the first stage enables to understand the behaviour of the channel and the critical conditions in the presence of noise and then a proper database is constructed under these conditions. Such database is exploited to develop an unsupervised algorithm, allowing to detect the presence of noise and classify it.

The reported numerical analyses have two main goals. The first consists of understanding the influence of different noise classes on the transmitted signal, drawing the conclusions about the properties of the naval environment under analysis. The second is the reliable identification and classification of the occurring noisy events. This aspect makes the simulation an effective tool to support the application of PLC technology in a transmission framework.

The paper is organized as follows. In Section 2 the models of the network and noises which have been analyzed are given; in Section 3 the unsupervised method for the classification of impulsive noises occurring in the channel is illustrated; in Section 4 numerical results and their discussions are displayed. Section 5 concludes the paper.

\section{Simulation Model}

In this section the simulation model for the evaluation of noise effects on the transmission of signals in a PLC line is presented. The simulation framework is composed by the circuit model of the powerline and the mathematical models of the injected noises. Details are given in the following two subsections.

2.1. Powerline Model. The powerline under analysis is modelled by a scheme, whose topology takes into account only the branches of the powerline connected to the most significant loads of the system. In detail, such scheme is composed by 70 branches, 13 ports, and 18 outlets, as sketched in Figure 1. This figure shows the position of the switch boards on the ship, the transmitter and the three receivers, named $\mathrm{Tx}, \mathrm{Rx}_{1}$, $\mathrm{Rx}_{2}$, and $\mathrm{Rx}_{3}$, respectively. The length of all the cables varies from a minimum of $6 \mathrm{~m}$ to a maximum of $60 \mathrm{~m}$. Such model can be exploited to provide a corresponding lumped circuit including information of both the characteristic impedance and the constants of the transmission line. In particular, it is supposed that each cable constitutes a uniform transmission line, whose characteristic impedance, propagation constant and length are denoted by $Z_{c}, \gamma$, and $l$, respectively. Hence, cables are modelled by an equivalent $\pi$-connection circuit, whose derived admittances $Y_{1}$ and $Y_{2}$ and the transversal one $Y_{3}$ are computed as:

$$
Y_{1}=\frac{\cosh (\gamma l)-1}{Z_{c} \sinh (\gamma l)}, \quad Y_{2}=Y_{1}, \quad Y_{3}=\frac{1}{Z_{c} \sinh (\gamma l)} .
$$

By applying expressions (1) to each cable of the network the lumped circuit can be obtained.

This lumped model can be successively exploited to simulate and test the general behaviour of the whole network. It allows to analyze the effects due to the presence of noise in any nodes. In fact, it is possible to simulate both a noise at source and an additive one by connecting a proper voltage generator to its injection point and to the receivers of the network, respectively.

2.2. Noise Model. In the examined case the presence of synchronous impulsive noises is considered. Such noises are generated when the most common appliances are disconnected or connected to the supply network. Basing on proper measurements in [13], the bursts associated to this kind of 


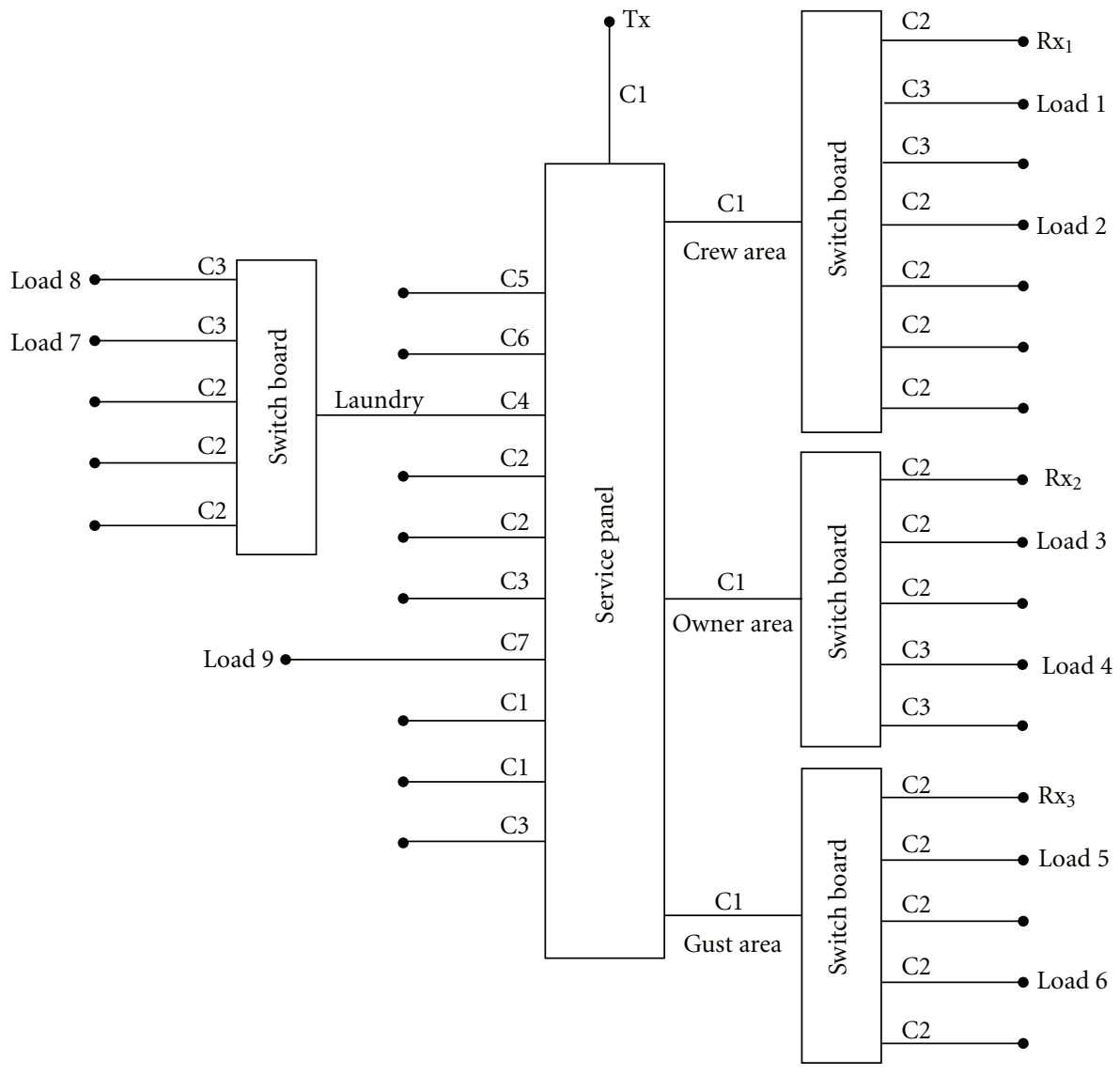

FIgURE 1: Topology of the in-ship network under analysis.

noise are modelled by two specific waveforms according to the duration of the event. In particular, a short burst can be seen as a dumped sinusoid, called elementary pulse, whereas a long burst is given by the succession of elementary pulses. The time realization of impulsive noise generated by the switches of appliances in a network can be modelled as:

$$
n(t)=A e^{-\alpha t} \cos \left(2 \pi k f_{0} t\right),
$$

with $A$ the amplitude of the pulse, $\alpha$ the damping coefficient, $k$ normally distributed random variable, $f_{0}$ frequency at which the load works, and $k f_{0}$ the pseudofrequency of the noise.

The values of the parameters $A$ and $\alpha$ can be determined by means of measurements carried out at source as shown in $[2,4]$, where six classes of impulsive noises are distinguished according to the kinds of appliances and events. In particular, classes 1 and 2 correspond to ON/OFF events of electrical switches and thermostats. Classes 3 and 4 are associated to puts and removals of plugs, respectively. Lastly, class 5 is due to starts of engines and class 6 collects different weak noises. In the cited papers amplitude and duration statistics about noises of each class are accurately measured and collected. Therefore, it is possible to exploit different realizations of the six classes of impulsive noises with the aim of analyzing the behaviour of the channel, as the injection point of noise and the receiver vary. In the considered simulations the noise realizations are modelled by proper voltage generators. In detail, the waveforms of such generators are given by the frequency spectra of the time realizations and are connected in series to the loads which could cause the occurrence of noise bursts.

Background noises are characterized by root mean square amplitudes which vary slowly over observation time. Although several probability density functions (PDFs) have been suggested in literature, the Nakagami- $m$ PDF seems to be the most effective in modelling background noise. Such function is given by the following expression [5]:

$$
p(r)=\frac{2}{\Gamma(m)}\left(\frac{m}{\Omega}\right)^{m} r^{2 m-1} e^{-m r^{2} / \Omega},
$$

$r$ being the random variable, $p$ the probability of the corresponding random variable, $\Gamma$ the Gamma function, $m$ the ratio of the moments, and $\Omega$ the mean power of the random variable. It is shown that this model is suited to represent different situations as the value of the parameter $m$ varies. In fact, if $m=1$, then a noise Rayleigh distributed is modelled, whereas if $m>1$ the Nakagami PDF fits noises with smaller variances and larger mean than the Rayleigh distributed one. If $m<1$ a noise with opposite characteristics is obtained. Due to its features, the reported model is considered the most general in the following with the aim of representing the background noise which takes place in naval environment. 
Impulsive noises are characterized by the rapid variations of their amplitudes.

For a sake of completeness of a realistic model of the analyzed network, asynchronous impulsive noise is considered. In fact, this kind of noise always occurs in PLC transmissions. Its most accepted model is given by Middleton's Class A one, according to which the amplitude $z_{n}$ is characterized by the following PDF [14]:

$$
p\left(z_{n}\right)=\sum_{m=0}^{\infty} \frac{e^{-A} A^{m}}{m !} \frac{1}{\sqrt{2 \pi} \sigma_{m}} e^{\left(-z^{2} / 2 \sigma_{m}^{2}\right)}
$$

with $\sigma_{m}^{2}=\sigma^{2}((m / A+\Gamma) /(1+\Gamma))$, being $p$ the probability of the corresponding random variable, $A$ a measure of the average number of impulses over a period of the signal, $\Gamma$ the ratio between the background noise power variance $\sigma_{b}$, and the impulsive noise power one $\sigma_{I}$, with $\sigma^{2}=\sigma_{b}^{2}+$ $\sigma_{I}^{2}$. Middleton's Class A model is based on the assumption that the total received noisy waveform consists of several sources each Poisson-distributed in time and space. This hypothesis is reasonable and in accordance with the model of the interarrival time between the occurring of two events [3].

\section{Proposed Classification Method}

In this section a method to detect and classify noises overlapped to the transmission of a signal in PLC systems is presented. To this aim, the signal, which is supposed to be collected at the receiver, can be modelled by the following expression:

$$
s_{k}(t)=x(t)+b(t)+n_{k}(t)
$$

with $k=1,2, \ldots, n_{r}$ and $n_{r}$ being the number of considered noise realizations, $x(t)$ the transmitted numerical signal, $b(t)$ the time realization of background noise, and $n_{k}(t)$ the time realization of a noise.

Let $\mathbf{S}$ be a set consisting of $n_{r}$ time signals $s_{k}(t), k=$ $1,2, \ldots, n_{r}$, given by the expression (4). The target of the proposed method is to partition the dataset $S$ in a number $c$ of different classes, each of which collects signals containing noisy contributions of the same kind. To this purpose, an unsupervised method requiring proper input data is considered. Data can be generally constituted either by the available signals or by features properly extracted from them. The former procedure implies the advantage of processing data without requiring any further elaborations; nevertheless the latter one enables to reduce data dimensionality and the redundancy of information that they contain, improving the performances of the classifier. As a consequence, the identification of proper features plays a fundamental role to classify the considered noises [15]. In the proposed method the features are, firstly, derived from the power density spectrum of signals at the receivers. Then, they are reduced in order to construct a dataset which is partitioned by making use of a self-organized map (SOM) as classifier.
In detail, let $S_{k}(f)$ be the frequency spectrum computed for each time realization $s_{k}(t)$, then the power density spectrum is denoted by $\left|S_{k}(f)\right|^{2}$. The vector $\mathbf{w}_{k}(f)$ is computed as

$$
\mathbf{w}_{k}(f)=20 \log \left(\left|S_{k}(f)\right|^{2}\right)-\widehat{S}_{k},
$$

$\hat{S}_{k}$ being the mean of the quantity $20 \log \left(\left|S_{k}(f)\right|^{2}\right)$.

The resulting vectors $\mathbf{w}_{k}(f), k=1,2, \ldots, n_{r}$ are arranged as the columns of a $\left(p \times n_{r}\right)$ matrix $\mathbf{X}$ which is decomposed by the technique of the principle component analysis (PCA). Such technique exploits the singular value decomposition to represent $\mathbf{X}$ as the matrix product:

$$
\mathbf{X}=\mathbf{U} \boldsymbol{\Sigma} \mathbf{V}^{T},
$$

where $\mathbf{U}$ and $\mathbf{V}$ are two unitary matrixes, whose dimensions are $p$-by- $p$ and $p$-by- $n_{r}$, respectively, and $\boldsymbol{\Sigma}$ is a $p$-by- $n_{r}$ diagonal matrix containing the generalized eigenvectors of $\mathbf{X}$.

The data space is reduced by taking into account the matrix $\mathbf{Y}$ which is obtained by projecting $\mathbf{X}$ into the space identified by its first $q$ singular vectors:

$$
\mathbf{Y}=\mathbf{U}_{q}^{T} \mathbf{X},
$$

$\mathbf{U}_{q}$ being the $q$-by- $q$ principal minor of $\mathbf{U}$.

The number of $q$ is determined as the minimum number of components assuring the maximum significant data variance. The columns of the resulting $q \times n_{r}$-matrix $\mathbf{Y}$ constitute the input dataset for a SOM.

This classifier is a particular kind of artificial neural network based on competitive learning, that is, its output neurons compete among themselves to be activated. In this way, only one output neuron or one group of neurons is updated when a datum is submitted to the net. In a SOM, the neurons are placed at the nodes of a grid. Neurons are selectively tuned to various input patterns or classes of them in the course of a competitive learning process. In particular, neurons characterized by the minimum distance from the input pattern are modified by the learning process. Therefore, the locations of the neurons become ordered with respect to each other in such a way that a meaningful coordinate system for different input features is created over the grid.

With the aim of identifying the unknown partition of dataset a SOM classifier searches $c$ centres, whose number is considerably greater than the expected one, in order to guarantee that every noisy event is recognized. This initial number can be established on the basis of an a priori knowledge or, if unavailable, by using the rule of thumb reported in [16]. This rule sets the initial number of centres to the square root of the data cardinality.

The number of the computed centres is, then, reduced in order to obtain the optimal landscape of centres. Such reduction is performed by eliminating those centres which are characterized by null clusters, since they are not representative of any datum. The final position of the neurons corresponding to the resulting elements is, subsequently, exploited to partition data into specific classes. In particular, a noisy event $N_{e}$ is considered overlapped to a signal $s(t)$ when among the neurons of the trained SOM one representing $N_{e}$ has the minimum distance from $s(t)$ in the space $\mathbf{Y}$. 
TABLE 1: Electric parameters modeling the channel under test.

\begin{tabular}{|c|c|c|c|c|c|c|}
\hline Cable & $f$ & $1 \mathrm{MHz}$ & $5 \mathrm{MHz}$ & $10 \mathrm{MHz}$ & $15 \mathrm{MHz}$ & $20 \mathrm{MHz}$ \\
\hline \multirow{3}{*}{$\mathrm{C} 1$} & $R(\Omega)$ & 0.064 & 0.18 & 0.19833 & 0.21 & 0.22 \\
\hline & $L(\mathrm{H})$ & $3.34 e-7$ & $3.29 e-7$ & $3.23 e-7$ & $3.29 e-7$ & $3.29 e-7$ \\
\hline & $C(\mathrm{~F})$ & & & $9.91 e-11$ & & \\
\hline \multirow{3}{*}{ C2 } & $R(\Omega)$ & 0.14 & 0.37 & 0.49 & 0.55 & 0.61 \\
\hline & $L(\mathrm{H})$ & $6.73 e-7$ & $6.62 e-7$ & $6.59 e-7$ & $6.58 e-7$ & $6.58 e-7$ \\
\hline & $C(\mathrm{~F})$ & & & $5.04 e-11$ & & \\
\hline \multirow{3}{*}{ C3 } & $R(\Omega)$ & 0.12 & 0.33 & 0.44 & 0.47 & 0.50 \\
\hline & $L(\mathrm{H})$ & $6.12 e-7$ & $6.03 e-7$ & $6.02 e-7$ & $6.01 e-7$ & $6.01 e-7$ \\
\hline & $C(\mathrm{~F})$ & & & $5.48 e-11$ & & \\
\hline \multirow{3}{*}{$\mathrm{C} 4$} & $R(\Omega)$ & 0.06 & 0.12 & 0.20 & 0.22 & 0.22 \\
\hline & $L(\mathrm{H})$ & $3.65 e-7$ & $3.63 e-7$ & $3.64 e-7$ & $3.64 e-7$ & $3.64 e-7$ \\
\hline & $C(\mathrm{~F})$ & & & $8.93 e-11$ & & \\
\hline \multirow{3}{*}{ C5 } & $R(\Omega)$ & 0.03 & 0.04 & 0.08 & 0.12 & 0.14 \\
\hline & $L(\mathrm{H})$ & $3.23 e-7$ & $3.22 e-7$ & $3.23 e-7$ & $3.25 e-7$ & $3.23 e-7$ \\
\hline & $C(\mathrm{~F})$ & & & $1.00 e-10$ & & \\
\hline \multirow{3}{*}{ C6 } & $R(\Omega)$ & 0.03 & 0.05 & 0.09 & 0.14 & 0.15 \\
\hline & $L(\mathrm{H})$ & $3.06 e-7$ & $3.05 e-7$ & $3.06 e-7$ & $3.06 e-7$ & $3.05 e-7$ \\
\hline & $C(\mathrm{~F})$ & & & $1.06 e-10$ & & \\
\hline \multirow{3}{*}{ C7 } & $R(\Omega)$ & 0.034 & 0.0641 & 0.11 & 0.16 & 0.17 \\
\hline & $L(\mathrm{H})$ & $3.07 e-7$ & $3.07 e-7$ & $3.07 e-7$ & $3.07 e-7$ & $3.07 e-7$ \\
\hline & $C(\mathrm{~F})$ & & & $1.06 e-10$ & & \\
\hline
\end{tabular}

TABLE 2: Load impedances in the channel under test $(\Omega)$.

\begin{tabular}{lccccccccc}
\hline Load & 1 & 2 & 3 & 4 & 5 & 6 & 7 & 8 & 9 \\
\hline$R$ & 75 & 22.89 & 26.56 & 75 & 14.34 & 75 & 32.04 & 9.52 & 0.64 \\
$x_{L}$ & $/ /$ & 0.26 & $/ /$ & $/ /$ & 0.16 & $/ /$ & 0.07 & 0.03 & 0.02 \\
\hline
\end{tabular}

\section{Simulation Tests and Results}

In the following two subsections, numerical results referring to the analysis of the channel are, firstly, provided and, then, the performances of the proposed classification method are given.

4.1. Frequency Domain Analysis of the Channel. The whole system has been simulated by using the circuit model reported in Section 2, whose electric parameters are summarized in Table 1, whereas the load impedances are given in Table 2. The simulations of the study case have been carried out by considering an impulsive input signal which is characterized by a spectrum having unitary amplitude in the frequency range of observation $(1 \mathrm{MHz}, 20 \mathrm{MHz})$. Such kind of signal is chosen to perform an analysis which is focused on the effects of noise on the response of the channel without taking into account the specific employed coding transmission.

In the conducted simulations the frequency spectrum of the voltages at receivers $\mathrm{Rx}_{1}, \mathrm{Rx}_{2}$, and $\mathrm{Rx}_{3}$ are computed injecting noise into each node to which the nine loads are connected. In detail, a time realization of each class of noise is produced and constitutes the waveform of a voltage generator which is connected in series to one of the loads. This analysis is carried out by considering the six classes of noise reported in Section 2. In Figure 2(a) the voltage at the receiver $\mathrm{Rx}_{1}$ is displayed when a noise belonging to class 1 is injected at nodes to which loads are connected. For a sake of clarity, in Figure 2(b) the same voltage spectrum curves are reported for the cases in which noise is injected into each node connected to an ohmic-inductive load. From an analysis of these figures, it can be noted that the noise having the greatest influence is injected at nodes to which resistive loads are connected. On the contrary, if the noise is injected into nodes with ohmic-inductive loads, five almost overlapped spectra are obtained. In other words, examining the obtained results, it can be deducted that ohmic-inductive loads attenuate the effects of noises. This is due to the fact that the modules of these impedances assume high values and, therefore, the most relevant part of the voltage which is supplied by the noisy generator localizes at the load.

Moreover, it should be considered that the curve with the highest values of voltage $V_{\mathrm{Rx} 1}$ in Figure 2(a) corresponds to a noise generated at the node with Load 1. It presents the maximum effects at the receiver. This behaviour is expected since this signal has to pass through the minimum path. This phenomenon can be understood by referring to the circuit model of the system in Figure 1. The topology has a star configuration, where the Service Panel is the star centre and connects all the other switchboards through paths formed by $\pi$-connections constituted of impedances with similar values. Consequently, the voltage at a receiver placed at a switch board does not see any loads placed at other switch boards or at the service panel. In an analogous way, 


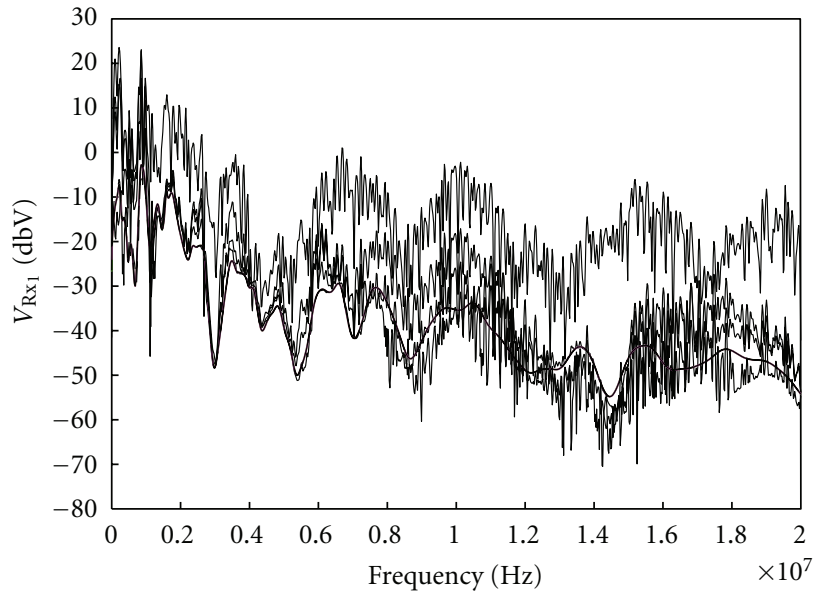

(a)

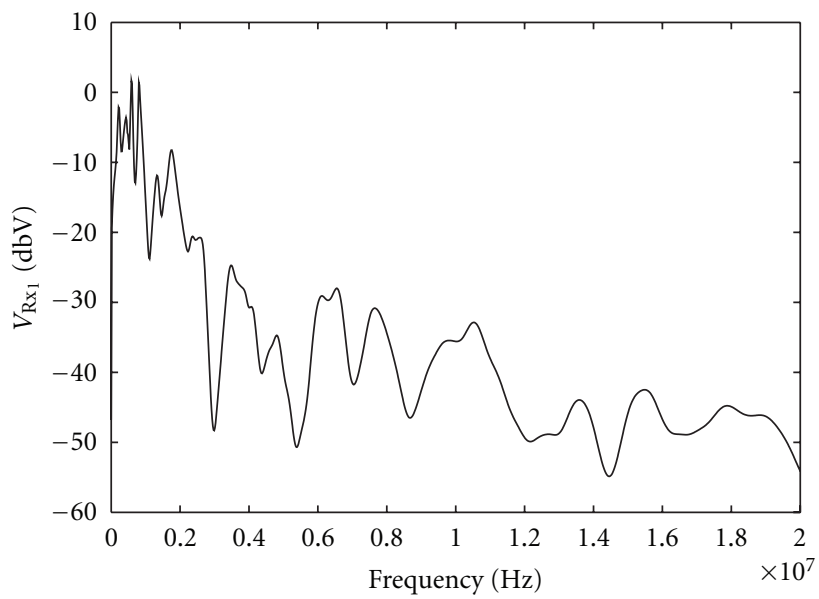

(b)

FIGURE 2: Spectrum of voltage at $\mathrm{Rx}_{1}$ when noise of class 1 is injected at (a) nodes with resistive loads and (b) with ohmic-inductive loads.

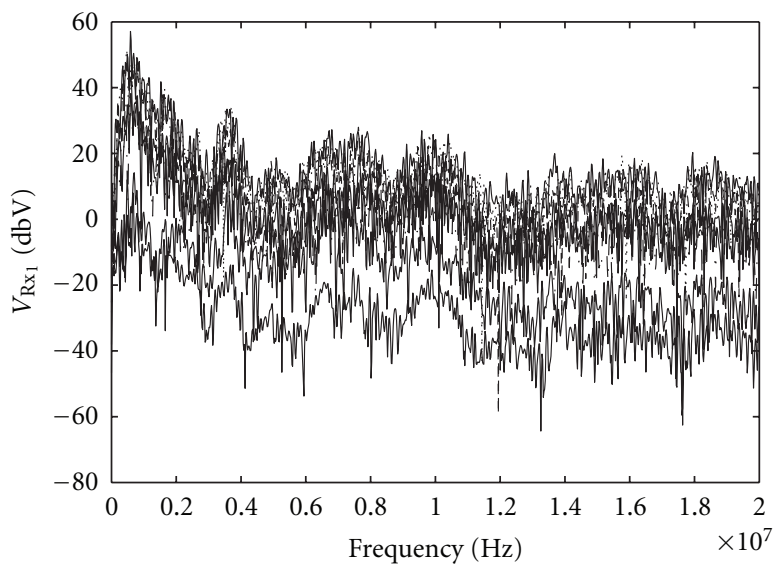

(a)

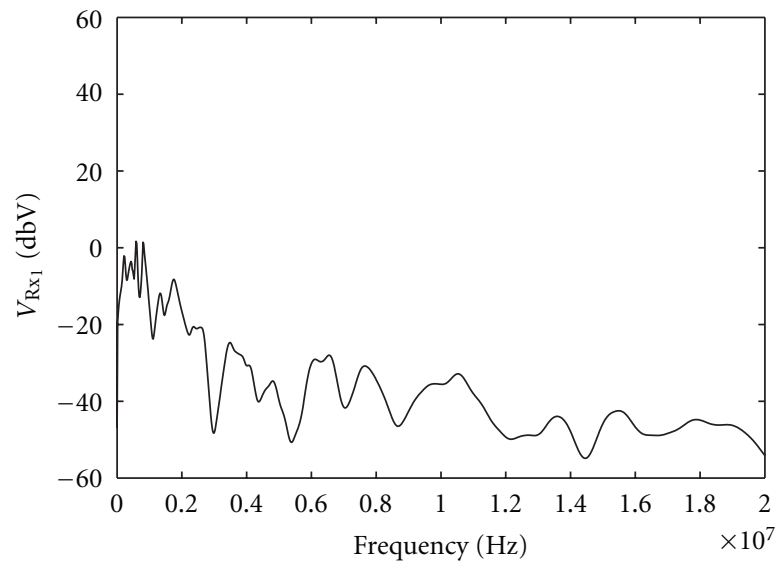

(b)

FIGURE 3: Spectrum of voltage at $\mathrm{Rx}_{1}$ when the six considered classes of noise are injected into node with (a) Load 1 and (b) Load 5.

the overall admittance at the star centre does not depend on load admittances placed at any node of the network. Therefore, the shape of the frequency responses exhibits little variations with respect to the variation of the loads. This behaviour can be considered general, in fact it has been observed at all receivers for each examined noise class, including the ones whose influence on the channel response is minimum [17]. It constitutes a novelty in PLC field and characterizes the naval applications [18].

In order to show this aspect, the effects of the different classes of noise at $\mathrm{Rx}_{1}$ have been considered. In Figures $3(\mathrm{a})$ and $3(\mathrm{~b})$ results are displayed by considering nodes with Loads 1 (resistive) and 5 (ohmic-inductive) as injection points for the six noises considered classes.

It can be evicted that previous behaviours are confirmed, in fact the maximum influence of noise is observed if loads are resistive and at the minimum distance from the receivers. In particular, the curves in Figure 3(a) reveal the maximum variability, whereas the spectra in Figure $3(\mathrm{~b})$ prove to be smoother than the ones given in Figure 3(a).
Finally, a proper simulation has been conducted. In such simulation, the signals at receivers are computed when noise is injected into nodes with resistive loads, whose impedances assume different values.

The case corresponding to the signal at receiver $\mathrm{Rx}_{1}$ when noise of class 1 is injected at Load 1 is reported in Figure 4 . In this case values of the load resistance equal to $75 \Omega, 1 \mathrm{k} \Omega$, and $100 \mathrm{k} \Omega$ have been considered yielding the three shown curves. It can be pointed out that curves become smoother at the increasing of the resistance, therefore the effect of noises reduces at the growing of this electric parameter.

4.2. Classification Performances. The information deriving from the previous tests have been exploited to implement the approach for the classification of impulsive events in Section 3. The simulations of the study case have been carried out by considering the generic waveform $s_{k}(t)$ given by the expression (3). In detail, the numerical input signal $x(t)$ is characterized by the parameters reported in 


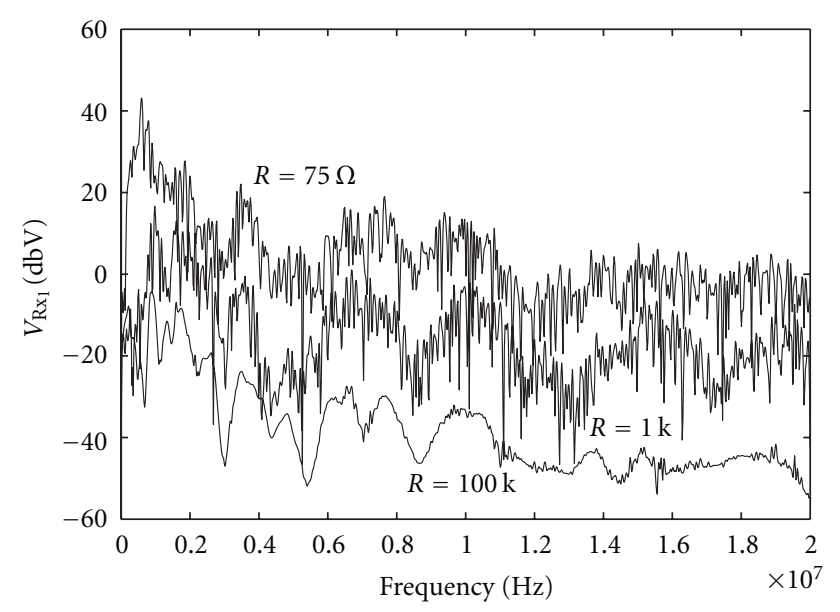

FIGURE 4: Spectrum of voltage at $\mathrm{Rx}_{1}$ in the presence of noise of class 1 when at Load 1 a resistor $R=75 \Omega, R=1 \mathrm{k} \Omega$, and $R=100 \mathrm{k} \Omega$ is connected.

TABLE 3: Features of a typical transmitted signal $x(t)$.

\begin{tabular}{lc}
\hline Frequency spectrum range & {$[1 \mathrm{MHz}, 7 \mathrm{MHz}]$} \\
Subcarrying band $B_{\mathrm{ch}}$ & $6 \mathrm{kHz} /$ subchannel \\
Sampling rate $f_{c}$ & $6 \mathrm{MHz}$ \\
Resampling rate $f_{c 1}$ & $24 \mathrm{MHz}$ \\
Modulation & $16-\mathrm{QAM}$ \\
Generated symbols & 16 \\
Guard Interval & $1 / 4$ \\
Symbol duration & $\left(1 / B_{\mathrm{ch}}\right) \times(1+$ Guard_Interval $)$ \\
\hline
\end{tabular}

Table 3. Such parameters are chosen to simulate a generic transmission of a signal in a wide band, like TV or similar ones.

The PDF of the background noise $b(t)$ is given by the Nakagami- $m$ function. For a sake of brevity such kind of noise is indicated as NBN in the following. The contribution $n_{k}(t)$ corresponds to a periodic synchronous impulsive distributed noise (PSIN) or to an asynchronous impulsive distributed noise (AIN) overlapped to the background.

The received signals are assured to be characterized by a signal to noise ratio of $30 \mathrm{~dB}$, as required by standard transmissions. In the reported study case $\mathrm{Rx}_{1}$ and node with Load 1 have been considered as receiver and injection point of synchronous impulsive noise, respectively. As shown in Section 4.1, this configuration has been chosen since it corresponds to a situation in which noise affects the transmission critically. The method has been tested by using a database formed by 240 time realizations for each kind of considered signal. In detail, the first 240 are obtained by overlapping as many as realizations of $b(t)$ to $x(t), 240$ realizations are obtained adding the realizations of the PSIN noise to the previous two signals, and 240 are obtained adding the realizations of the AIN noise to $b(t)$ and $x(t)$ signals. Finally, it should be remarked that each class of the periodic synchronous impulsive noise is represented by 40 realizations. This dataset has been subsequently split in two subsets

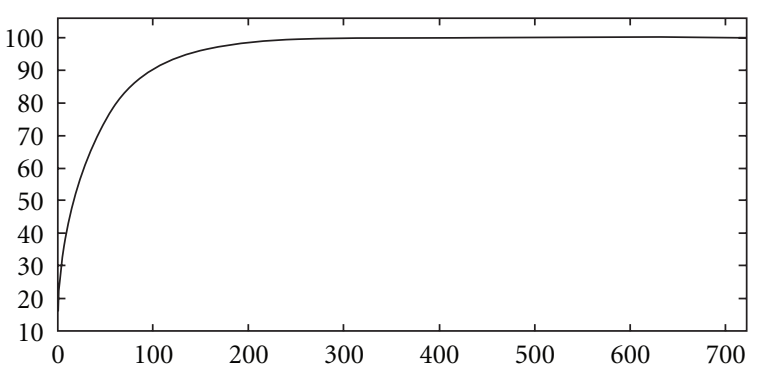

Figure 5: Pareto diagram of the PCA components of a signal belonging to the set of the considered ones.

consisting in the $80 \%$ and $20 \%$ of the initial data. They are used for training and testing the SOM. The classification has been performed using a 4-by-4 SOM, since it allows to reach the best performances during the training phase in all the considered cases. A number $q=19$ PCA components of the obtained signals has been considered. As it can be observed in Figure 5, this number of components corresponds to the $50 \%$ of Pareto diagram, which reports the variances of principal components versus their number. The choice of $50 \%$ in this diagram guarantees a good trade-off between the computational time and the correct identification.

The performances of the proposed detection method have been evaluated by defining the correct classification index CC as:

$$
\mathrm{CC}=\frac{\text { Number of events correctly detected }}{\text { Total number of events }} \times 100 .
$$

A given noisy event is considered as correctly classified if a waveform is assigned to the set associated to the class of noise overlapped to the transmitted signal.

Different tests have been conducted with the aim of understanding the capabilities of the proposed method to classify different noisy conditions.

The first experiment consists of distinguishing two classes: the former is given by a Nakagami distributed background, whereas the latter corresponds to a PSIN distributed noise overlapped to the background. In other words, this experiment aims at detecting the presence of an impulsive event during the data transmission. In this case a value of the CC index equal to $100 \%$ has been obtained if considering each of the six PSIN classes overlapped to $x(t)$. The same result is obtained for all the experimented values of $m$.

The second experiment has been performed in order to show the sensitivity of the method to the different classes of noise in a more realistic situation. In particular, it has been considered the presence of the asynchronous impulsive noise (AIN) which commonly affects PLC transmissions. To this aim, the realizations of this kind of impulsive noise have been generated by Middleton's PDF. The database described for the first experiment has been enlarged by considering further 240 signals. As a consequence, three situations can be identified: the absence of impulsive noises, the presence of the PSIN noise, and the presence of the asynchronous impulsive one. The values of the CC index are obtained when the presence of only one class of PSIN is considered. Table 4 
TABle 4: CC index obtained in the detection of Nakagami, Middleton, and one class of PSIN noises $(m=1)$.

\begin{tabular}{lccc}
\hline PSIN class & NBN & NBN + AIN [\%] & NBN + PSIN [\%] \\
\hline 1 & $100 \%$ & $100 \%$ & $100 \%$ \\
2 & $100 \%$ & $100 \%$ & $100 \%$ \\
3 & $97.91 \%$ & $100 \%$ & $100 \%$ \\
4 & $100 \%$ & $100 \%$ & $100 \%$ \\
5 & $100 \%$ & $97.91 \%$ & $100 \%$ \\
6 & $100 \%$ & $100 \%$ & $100 \%$ \\
\hline
\end{tabular}

TABle 5: CC index obtained in the detection of Nakagami, Middleton, and PSIN noises as the value of $m$ varies.

\begin{tabular}{lccc}
\hline & NBN & NBN + AIN & NBN + PSIN \\
\hline$m=1$ & $99.47 \%$ & $96.87 \%$ & $100 \%$ \\
$m=2$ & $100 \%$ & $100 \%$ & $100 \%$ \\
$m=3$ & $100 \%$ & $99.47 \%$ & $100 \%$ \\
\hline
\end{tabular}

reports the results for the test set corresponding to $m=1$, of the Nakagami PDF.

From an analysis of this table, it can be observed that the synchronous impulsive noise is detected without errors, whereas misclassifications occur for the asynchronous and background noises. Therefore it is possible to assert that the performances do not decay if a specific class of noise affect the electric system. Results are consistent, in fact the method does not change its classification capabilities as the kinds of noise vary.

The third experiment is devoted to evaluate the robustness of the classification method as the background noise level changes. In this case, the different kinds of PSINs realizations are grouped in only one class. Therefore, the presence of AINs, PSINs, or the absence of impulsive noises is detected as the values of parameter $m$ change. In Table 5 the results corresponding to $m=1,2$, and 3 of the Nakagami PDF are reported.

It should be noted that the method exhibits good performances in terms of correct classification in almost all the considered cases as the power of the background noise increases.

With the aim of evaluating the capabilities of the approach to classify correctly noisy events under different operating conditions, the values of CC have been computed by assuming that the classes of noises used in the train phase and the ones classified at the receiver are different. As supposed in previous cases noise realizations belonging to all the classes of PSIN compose a unique set. The results of this last experiment are reported in Table 6. The first column contains the classes used to train the SOM. This set changes for each considered case. On the contrary, the number of the classes to be recognized is constant and equal to 3 , as reported in the first row. The results shown in Table 6 prove that the method enables to detect and classify impulsive noises with good accuracy under different operating conditions. In fact, a class is not identified in the classification stage if it is not employed in the training set. Finally, it should be observed
TABLE 6: CC index obtained in the detection of Nakagami, Middleton, and PSIN noises with different training sets.

\begin{tabular}{lccc}
\hline Training classes & NBN & $\begin{array}{c}\text { Recognized classes } \\
\text { NBN + AIN }\end{array}$ & NBN + PSIN \\
\hline NBN; & $100 \%$ & $97.91 \%$ & $100 \%$ \\
NBN + AIN & & & \\
NBN + PSIN & $100 \%$ & $100 \%$ & 0 \\
\hline NBN; & $100 \%$ & 0 & $95.80 \%$ \\
\hline NBN + AIN & NBN + PSIN & & \\
\hline
\end{tabular}

that two values of CC are not $100 \%$. This is due to the fact that some impulsive events can be misclassified with the background noise class, if high values do not characterize them. Nevertheless, this behaviour can be expected. In fact, the first row of Table 6 is coherent with the results in Table 4, whereas the last row is caused by the fact that the classifier is forced to recognize a number of classes which varies from the train to the test.

\section{Conclusions}

In this paper, a numerical analysis of the effects of synchronous periodic impulsive noise on the transmission of wideband signals in a naval PLC has been conducted. Six classes of this impulsive noise modelled at source have been considered to affect the system. These kinds of noises simulate the presence of different common appliances that change their status from OFF to ON and vice versa. The conducted simulations show that the kind of loads and the position of the injection point in the topology of the network are the parameters that mainly influence the properties of the PLC transmission. In particular, such systems are characterized by electric plants, whose topology minimizes the effects of the transfer function variations caused by switching loads. Therefore, the classification of noisy events plays a fundamental role in this field, since the presence of noise is the main factor which prohibits the full application of PLC. To this aim, an unsupervised technique based on the use of PCA and a SOM classifier has been developed.

Three contributions of noise which commonly verify in PLC naval systems have been considered: the first is given by the background, whereas the second and the third correspond to a periodic asynchronous and synchronous impulsive noise overlapped to the background. Numerical results show that the proposed approach enables to classify the typologies of noise accurately under different operating conditions. Therefore, the developed method represents a useful tool for the detection and classification of noisy events in this kind of transmission.

Future analyses will be devoted to investigate the effects of further kinds of noises which could affect the channel under test and the effectiveness of the proposed solution in their detection. 


\section{Acknowledgment}

This work has been supported by Ministero dell'Istruzione dell’Università e della Ricerca under Project PRIN 2007.

\section{References}

[1] N. Andreadou and F. N. Pavlidou, "PLC channel: impulsive noise modelling and its performance evaluation under different array coding schemes," IEEE Transactions on Power Delivery, vol. 24, no. 2, pp. 585-595, 2009.

[2] M. Tlich, H. Chaouche, A. Zeddam, and P. Pagani, "Novel approach for PLC impulsive noise modelling," in Proceedings of IEEE International Symposium on Power Line Communications and its Applications (ISPLC '09), pp. 20-25, Dresden, Germany, April 2009.

[3] M. Zimmermann and K. Dostert, "Analysis and modeling of impulsive noise in broad-band powerline communications," IEEE Transactions on Electromagnetic Compatibility, vol. 44, no. 1, pp. 249-258, 2002.

[4] M. Tlich, H. Chaouche, A. Zeddam, and F. Gauthier, "Impulsive noise characterization at source," in Proceedings of the IFIP Wireless Days Conference, 2nd International Home Networking (IHN '08), Dubai, United Arab Emirates, November 2008.

[5] H. Meng, Y. L. Guan, and S. Chen, "Modeling and analysis of noise effects on broadband power-line communications," IEEE Transactions on Power Delivery, vol. 20, no. 2, pp. 630637, 2005.

[6] M. Katayama, T. Yamazato, and H. Okada, "A mathematical model of noise in narrowband power line communication systems," IEEE Journal on Selected Areas in Communications, vol. 24, no. 7, pp. 1267-1276, 2006.

[7] O. G. Hooijen, "A channel model for the residential power circuit used as a digital communications medium," IEEE Transactions on Electromagnetic Compatibility, vol. 40, no. 4, pp. 331-336, 1998.

[8] D. P. Filev, R. B. Chinnam, F. Tseng, and P. Baruah, "An industrial strength novelty detection framework for autonomous equipment monitoring and diagnostics," IEEE Transactions on Industrial Informatics, vol. 6, no. 4, pp. 767-779, 2010.

[9] G. Laguna and R. Barrón, "Survey on indoor power line communication channel modeling," in Proceedings of the 5th Meeting of the Electronics, Robotics and Automotive Mechanics Conference (CERMA '08), pp. 163-168, Cuernavaca, Morelos, Mexico, October 2008.

[10] N. Pavlidou, A. J. Han Vinck, J. Yazdani, and B. Honary, "Power line communications: state of the art and future trends," IEEE Communications Magazine, vol. 41, no. 4, pp. 34-40, 2003.

[11] S. Tsuzuki, M. Yoshida, Y. Yamada et al., "Characteristics of power-line channels in cargo ships," in Proceedings of IEEE International Symposium on Power Line Communications and Its Applications (ISPLC '07), pp. 324-329, Pisa, Italy, March 2007.

[12] J. Nishioka, S. Tsuzuki, M. Yoshida, H. Kawasaki, T. Shinpo, and Y. Yamada, "Characteristics of $440 \mathrm{~V}$ power-line channels in container ships," in Proceedings of IEEE International Symposium on Power Line Communications and its Applications (ISPLC'09), pp. 217-222, Dresden, Germany, April 2009.

[13] V. Degardin, M. Lienard, A. Zeddam, F. Gauthier, and P. Degauque, "Classification and characterization of impulsive noise on indoor power line used for data communications,"
IEEE Transactions on Consumer Electronics, vol. 48, no. 4, pp. 913-918, 2002.

[14] W. Bo, Q. Yanghao, H. Peiwei, and C. Wenhao, "Indoor powerline channel simulation and capacity analysis," in Proceedings of the IET Conference on Wireless, Mobile and Sensor Networks (CCWMSN '07), pp. 154-157, Shanghai, China, December 2007.

[15] J. H. Zhou, C. K. Pang, F. L. Lewis, and Z. W. Zhong, "Intelligent diagnosis and prognosis of tool wear using dominant feature identification," IEEE Transactions on Industrial Informatics, vol. 5, no. 4, pp. 454-464, 2009.

[16] S. Yu and K. Zhu, "Optimum coordinate number of clusters and best clustering in fuzzy C- means," in Proceedings of the 7th World Congress on Intelligent Control and Automation (WCICA '08), pp. 5776-5781, Chongqing, China, June 2008.

[17] G. Acciani, V. Amoruso, G. Fornarelli, and A. Giaquinto, "Numerical analysis of synchronous impulsive noise on naval powerline communications," in Proceedings of IEEE International Symposium on Industrial Electronics (ISIE '10), pp. 29732978, Bari, Italy, July 2010.

[18] T. Zheng, M. Raugi, and M. Tucci, "Analysis of transmission properties of naval power line channels," in Proceedings of IEEE International Symposium on Industrial Electronics (ISIE '10), pp. 2955-2960, Bari, Italy, July 2010. 

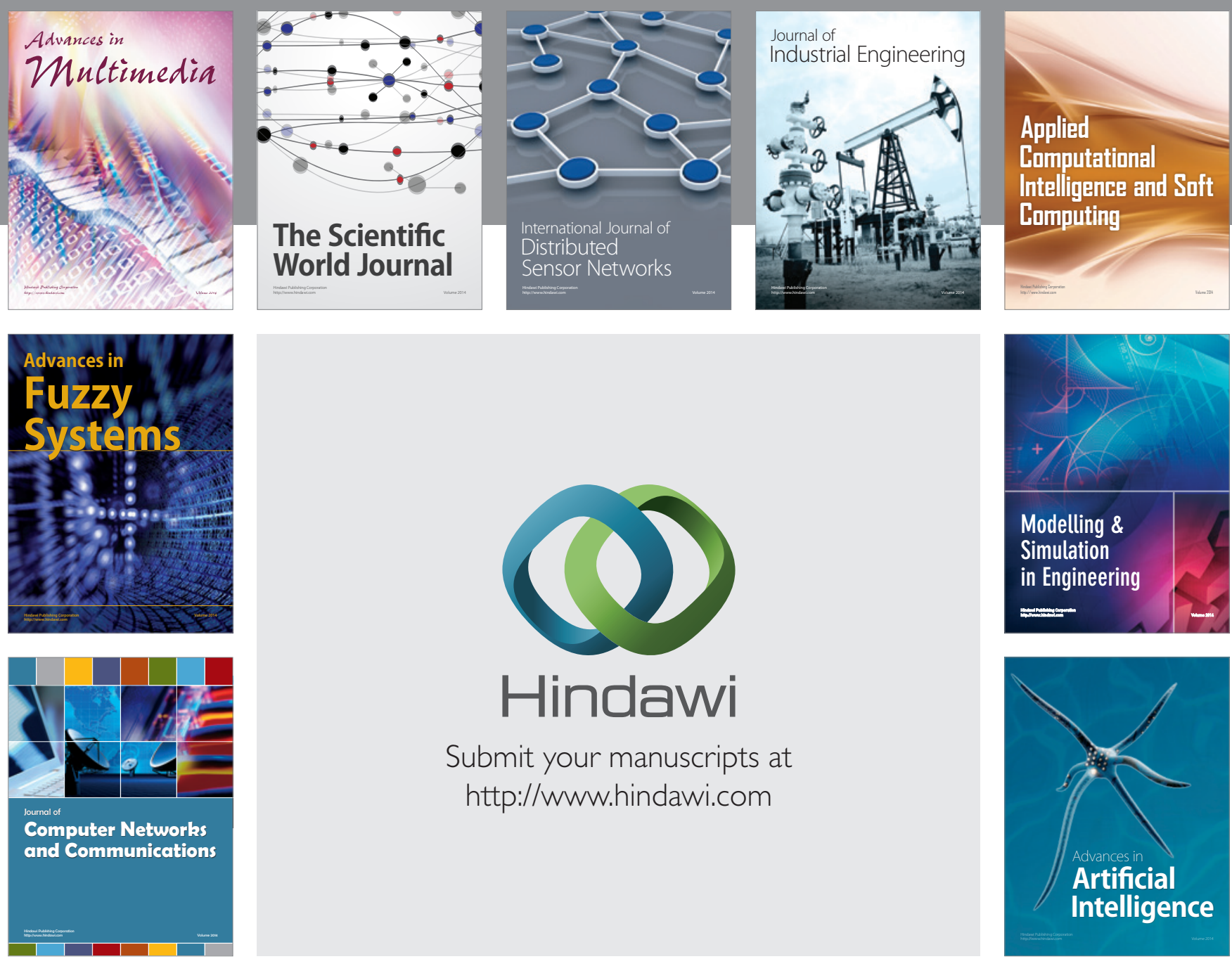

Submit your manuscripts at

http://www.hindawi.com
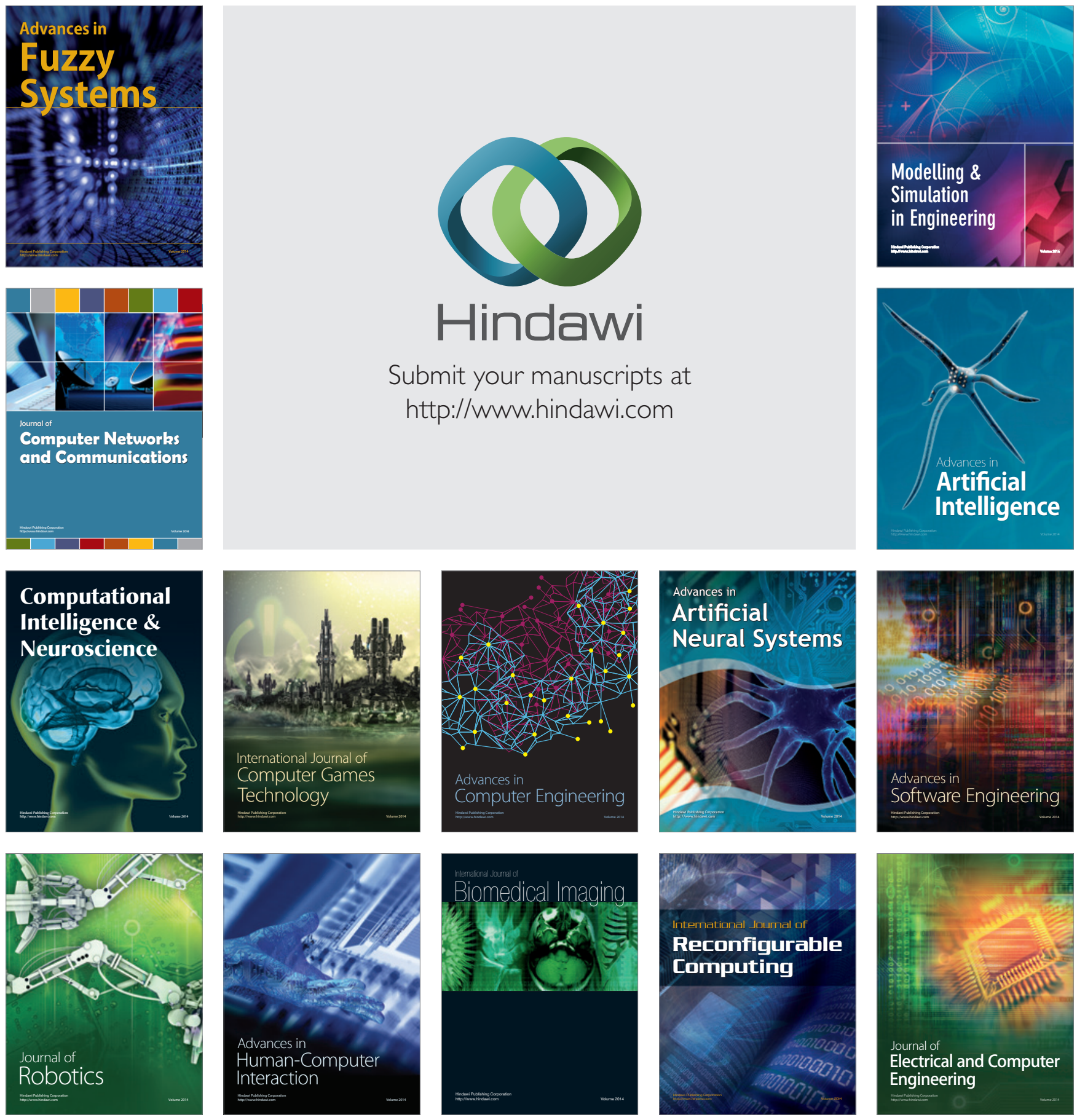